\title{
The Epistemological Crisis in Physical Education: Implications for Teaching Work
}

\author{
By Claudio Pellini Vargas* \\ Antonio Flavio Barbosa Moreira ${ }^{+}$
}

\begin{abstract}
This paper analyzes the crisis in Physical Education by considering it historically in relation to the crisis in Modernity itself. Firstly, we highlight the object of conflict between mind and body (reason and sensitivity) by pointing out this dichotomy as the first cause for this crisis. Secondly, we distinguish an "oblatu" from a "transfuga", based on Ricardo Vieira's considerations, by applying this distinction to the mind/body dichotomy. Thirdly, we emphasize the importance of teaching to a more profound process in a Physical Education teacher's education. Finally, we present and analyze, based on our academic and professional experiences, examples of real teaching situations and associate them to a lack of epistemological identity affecting the teaching profession, as well as the perspective for an "oblatu" and a "transfuga". We conclude by highlighting challenges that must be overcome by Physical Education teachers in the continuous process of (self) education and insist on the view that a greater understanding of teaching could encourage this process.

Keywords: Physical Education; Teachers; Teacher's Work; Identity
\end{abstract}

\section{Initial Considerations}

In 1882, Rui Barbosa gave his opinion on Project 224 - the Leôncio de Carvalho Reform, Decree No. 7247, April 19, 1879, of the Public Statement, in which he defended the inclusion of Physical Education in schools and the equivalence of Physical Education teachers to those from other disciplines. In that report, he pointed out and explained his ideas about the importance of having a healthy body to sustain the intellectual activity. (BRAZIL, 1997, emphasis added)

In accordance with the rationality that is typical of modern times, the State undertook the task of educating children and adolescents, as well as "softening" their behavior. In order to achieve that goal the role played by educational institutions was particularly emphasized. Over the centuries - especially in Modern times - punitive and coercive legislations were created in various

${ }^{*}$ Ph.D. Student, Federal University of Juiz de for a, Brazil.

${ }^{+}$Emeritus Professor, Federal University of Rio de Janeiro, Brazil and Full Professor, Catholic University of Petrópolis, Brazil. 
ways. Each period of time created its own system of arbitrary laws to establish teaching, domesticate human bodies and ensure private and public rights by punishing those who were not suited to the established order. For Foucault (2004), the use of punitive methods was meant to re-adapt the individual to society and prepare his/her body to properly exert the appropriate activities.

Since the emergence of the Enlightenment, individual emancipation was essentially seen as a process of rationality. The body needed only be tamed. At that time, an individual, seen as centered and unified (HALL, 2006), would only have his/her right to inclusion and/or belonging to society recognized if his/her reason had developed. This individual was practically limited to rationality.

This perspective is reflected even in contemporary times, in the appreciation of various theoretical currents of development and transmission of knowledge (constructivism, social constructivism, information processing theories) which focus on cognition by expressing that the fundamental way to learn takes place only through reason. Over the $19^{\text {th }}$ and $20^{\text {th }}$ centuries, the scientific methodology through which such strands come together made it difficult for "new teaching and learning possibilities" to exist by using other logics that would consider intuition, emotion or corporeality. Body - in the sense of expressing one's "will, desire, sensitivity, instinct" - was considered as a barrier to "truth", which had shifted from Theology and Philosophy towards Science since the beginning of modern thought.

In this context, the body corresponded to a single object or "machine", activated by a centralizing reason. The constant search for order, typical of modernity, would hinder bodily expressions, intuitions, instincts and/or desires. According to Bracht (1999, p. 71), the "role of corporeality in learning has been historically undervalued, overlooked [...]. The deficit in body dignity would come from its secondary aspect before the emancipating force of spirit or reason." The appreciation of body is not harmonized with the intentions of rationality. However, the principles underlying this appreciation, i.e., the privilege granted to reason, are now widely destabilized. In other words, we can actually speak of a crisis in rationality in the context of a crisis in Modernity.

When we question the privilege granted to rationality, we challenge the mind/body dichotomy. This questioning reaches Physical Education (PE) in school, which is also affected by this crisis (BRACHT, 2007). We argue that $\mathrm{PE}$ is going through an identity crisis, which strongly destabilizes the epistemological identity of a worker, making him/her oscillate in the construction of his/her personal and social identity, between the identities of the oblatu and the transfuga (VIEIRA, 2009), which are subsequently characterized without being able to structure their experiences in order to promote a more autonomous independent learning. Finally, we argue that the study of situations experienced in the course of teaching can be an important strategy to allow the PE teacher to reflect on his/her practice and become a subject in his/her training process. Thus, we reversed the direction usually found in many analyses which goes from education to teaching. We highlight 
the important contribution that the increased understanding of features, details and nuances of this work can offer to the teacher education process.

In order to discuss this subject, we emphasize the conflict between reason and body (rationality and sensitivity) in the text, in an attempt to show that such a dichotomy is found in the roots of the crisis. Next, we distinguish the oblatu from the transfuga based on Vieira's considerations (2009) by applying this distinction to the mind/body dichotomy, which we positioned as a point of emergence from the crisis. Thirdly, we emphasize the importance of a teacher's work for a deeper process of education. Finally, we show examples of situations of a teacher's work based on our academic and professional experiences, relating them to a lack of epistemological identity that defines PE and its practice today, as well as the prospects for an oblatu and a transfuga. We conclude by highlighting challenges that must be overcome by PE teachers in a continuous self-education process and advocate the viewpoint from which a greater understanding of teaching can facilitate such process.

\section{The Physical Education Discipline and its Epistemological Identity Crisis}

According to Bracht (1999, p. 72), the concept of "discipline" has an ambiguous meaning. If, on the one hand, the term presents "the aspects of knowledge itself", on the other hand, it shows the presence of "hierarchical relationships, the observance of precepts and rules of conduct for the body". In this second approach, according to Foucault,

Discipline produces [...] submissive, exercised, "docile" bodies. Discipline increases the body forces (in economic terms of use) and decreases those same forces (in political terms of obedience). [...] It decouples power from the body; (2004, p. 119).

The historical mind/body dichotomy encourages us to think that both "parts" of this division should be valued. Thus, the modern and rationalist thought in its eagerness for the pursuit of order and control suggests that school would be one of the institutions capable of promoting a "formal" possibility to educate mind and body. On the one hand, intellectualized (cognitive) education; on the other hand, body (physical) education. Such a separation leads to the following question, which is central to this work: How could we think of PE in epistemological terms (as a science) when body and motion - its objects of study - are not linked to cognition? To deal with this issue, Bracht (2007, p. 30-31) states that "a bit of the PE identity crisis comes from here, the desire to become a science, and from the finding that it depends on other scientific disciplines (Physical Education is epistemologically "colonized" by other disciplines)."

On the PE level, its multiple subfields do not seem to find a common denominator, in the sense that the discipline cannot offer an epistemological 
identity to its professionals. Bracht (2007, p. 32) defines epistemological identity as "the unique shape through which each scientific discipline questions and explains reality, which is determined by the type of problem raised by it, by research methods and by the language it developed and uses".

The worker seems to look for this identification (with science) in other branches of knowledge, pressed by insecurity and by a desire for recognition which, in his/her opinion, his/her field of education does not and maybe will never offer. Thus, there are many experts in Physiology, Sports Psychology, Sports Sociology etc., but few in Physical Education. Additionally, the pedagogical practice of $\mathrm{PE}$ does not seem to be a concern for the abovementioned subfields. Bracht says:

... the academic field of $P E$ is fragmented; the scientific languages that are spoken are differentiated, specific. [...] experts have emerged, not in PE, but in Exercise Physiology, Biomechanics, Sports Psychology, etc. [...] Because of the specialization process it was not very long before a "dialogue of the deaf" was implemented. (2007, p. 31)

In this context, we then ask: Is PE a science? Bracht (2007, p. 32) radicalizes and argues "that PE is not a science". But we ask: Why does this question bother so many professionals? The answer seems to be attributed to the status that science has in contemporary times, because any discipline, in any field, needs and seeks to take a scientific status. PE teachers do not want to lose such a prominent position. The traditional hegemonic PE is an offspring of Modernity (BRACHT, 2007), i.e., it was developed at a time when rationale consisted of the correct and only way to interpret the real world. While it suffered intense influence from the objective scientific thought, it failed to correctly respond to the expectations of such intellectual pressure.

In summary, the epistemological crisis of PE corresponds to the difficulty in considering it a discipline, since it does not provide the necessary features to be regarded as a science. We argue that this uncertainty can still be seen today, despite all questioning ever made to scientific knowledge, and is no longer seen as being able to "reflect" physical and social reality.

\section{The Oblatu and the Transfuga: The Passage "from" and "between" Cultures/Social Groups}

In addition to the epistemological identity crisis in PE, which so severely affects the professional teacher identity, it is important to note the crisis he/she is faced with due to the transformations which the process of construction of personal and social identities has gone through, in which an individual assumes different identities in different moments - identities that are not necessarily unified around a coherent "Me". This argument is based on Ricardo Vieira 
(2009), for whom the construction of identities is a complex dialectic process, or a permanent, flexible and dynamic reconstruction.

This reconstruction of personal and social identity is intrinsic to every individual, and does not constitute a mere reproduction of the social and cultural sphere within which it moves. Individuals jump from one social group to another, from one situation to another, from one society to another, from one domain of existence to another, without there necessarily being any continuity, homogeneity and compatibility between all these experiences. According to the types of passages that may occur, Vieira (2009) distinguishes the oblatu from the transfuga.

The former acquires a new educational cultural outlook when they associate to a new social group, leaving the other group whose values they start to reject. The individual is re-educated, assimilates and absorbs the values of the new culture. He/she actually gives the impression that he/she has never known any other way of seeing and being in the world. A true metamorphosis then ensues with the products of the new culture.

The transfuga is the one who, despite accepting and seizing upon a new culture, does not reject the culture of origin. He builds attitude and contextualizing bridges between the cultural spheres he/she crosses. The culture of origin then receives a new dimension, but is neither rejected nor annihilated. The transfuga becomes a new "other" from the new others who dwell the new cultural space, without renouncing the previous "others" who had already been incorporated and have significant importance for the new "outlook" that is to be worn.

For an oblatu, a river separates both cultures, and there is no continuity between them. The transfuga, on the other hand, despite receiving and accepting the new culture, does not reject his/her culture of origin. Vieira adds that syncretism arises from the dialogue between that which has already been acquired and that which is now acquired corresponding to the emergence of a new dimension of being - the third one - non-static, never finished, always subject to change and to reconstructions of identity. This is the "instructed third", which results from the dynamic and unfinished integration of a certain cultural and social background and new values bound by the new cultural riverbank. The instructed third builds the bridge between the two (or more) banks, and this bridge becomes part of his/her new identity. The transfuga departs, but remains there, still in both spaces and, perhaps in a third space, which corresponds to the bridge itself, built by him/her, connecting these cultural territories.

In contemporary times, the consequences of globalization influence the changes in identity due to the dialectics of the global and the local. The identity takes new contours, which are characterized by uncertainty, instability, inaccuracy, discontinuity. Identity then is permanently constructed (Vieira, 2009).

In this context, individuals are inevitably researchers of themselves. Without a personal reflection, adds Vieira, there is no education. This 
education consists of providing other human beings with the tools they need to structure their experience so that they can "continually extend knowledge, rational belief, understanding, autonomy, authenticity, and a sense of the proper situation in the past, present and future of human beings" (VIEIRA, 2009, p. 68). Thus, educating is transforming, i.e., educating is leading one to want to transform him/herself.

In other words, educating is giving the individual the means to structure his/her experience in order to help expand what he/she knows, what he/she believes in, and what he/she doubts. It consists of providing the means to gain access to knowledge, understanding, as well as to continue to expand them. Vieira adds:

... education for trainers and teachers must have a simultaneously anthropological and ecological dimension, able to increasingly encourage comparative thinking, reflective thinking, comprehensive thinking, cultural relativism, integration of the local and the global in learning. (2009, p. 70)

We suggest that the arguments presented by Vieira can be enriched by including in the education process that which Bauman (2008) called third degree learning. For the Polish sociologist, learning to learn, as highlighted by Vieira, is an inevitable complement of the so-called first degree learning, which in turn is associated with the transmission of fragmented knowledge that usually makes up a curriculum. To Bauman, however, second degree (continuing to learn) learning retains its value and works as expected only when students believe that the contingencies they may find will match a stable pattern. It is this learning that can be linked to learning ability. Thus, we ask: Will PE teachers find stable conditions in our schools? How could we then conceive the ability to learn?

According to Bauman (2008), each one of the orientation axes that have made the world seem solid and have guided the selection of certain life strategies (jobs, skills, practice, human partnerships, models of property and propriety, visions of health and disease, values to be pursued and the means to pursue them) is shown to be in permanent flow. According to the author, third degree learning, which is inevitable today, corresponds to a type of learning our educational institutions are not yet prepared to offer. It is a kind of learning that is considered by modern educational theory as a pathological phenomenon or an advanced schizophrenic attack. Third degree learning occurs, as clarified by Bauman, when a student acquires the skills to modify the set of alternatives that he/she learned to consider and deal with in the course of the learning-tolearn process. In this context, there is no reason why we should worry about the issue of standards: the type of habit to be acquired is the habit of living with no habits (Bauman, 2008). In other words, the learning-to-learn process needs to become flexible and open up to changes and uncertainties that necessarily mark living and socializing in pure Modernity. 
Let us return to the oblatu and the transfuga and Vieira's education view (2009). We suggest that in the practice of PE an oblatu may correspond to a teacher who, while appreciating rationality and science, crosses the margin into and accommodates in corporeality, and rejects and abandons those values and practices associated with the culture of origin - modern rationality. The effort to relegate his/her initial adherence to a forgotten corner responds both through its metamorphosis and the insecurity derived from a transformation that can never be total, however much he seeks to keep "both banks of the river" separated. It is this perspective that, in our view, is still evident in many practices and experiences of PE teaching.

Transfugas, on the other hand, are able to gather multiple elements - of rationality and corporeality -, align, mix, and tangle them up in different ways, in different situations, without belittling or denying any of them. In this process, we must highlight the constant self-reflection attitude and questioning. Thus, transfugas become products of the different cultures they cross and the different cultures that cross them, building professional, personal and social identities marked by hybridism. These are the teachers whose self-education we defend. They are the ones who may more easily acquire the skills to better structure their experiences, learn to learn, increase their knowledge and understanding of themselves, of the others and the world, as well as suspect all these abilities and look for alternatives in the modern net world to provide other routes, other experiences, other values and other forms of relationship and dialogue. They are the ones who may approach third degree learning as proposed by Bauman.

Next, we focus on excerpts from teaching through reports on remarkable situations that we encountered throughout our professional practice and our academic and school experiences, particularly in PE. Such facts occurred in different cities and are reported with due care to ensure that the required ethical limits and anonymity are preserved. We believe such reports are complementary and highlight the identity crisis in this field, as well as the perspective (oblatu and transfuga) taken by the teacher in the process of rebuilding his/her identity.

\section{Implications for Teaching in Physical Education}

It is important to make it clear that we understand teaching based on Assunção and Oliveira, who see it as follows:

... the teaching category encompasses both the individuals in their complex dimensions, experiences and identities and the conditions under which activities are carried out in the school setting. It includes, therefore, activities, responsibilities, and relationships that take place at school apart from class management and are subject, 
as a whole, to those mechanisms deployed by the management in the search for lower costs and increased efficiency. (2009, p. 353)

Let us, then, move on to reports concerning individuals, conditions, activities, responsibilities and relationships which are to be found in different PE practices.

The first report is one of the ambivalent speeches by a PE teacher during an interview in recent research (Vargas, 2010, p. 43). This speech seems to demonstrate a deep confusion on the part of the respondent regarding the title he holds:

Professor Rafael: I had very good teachers in the Pedagogical Area of Physical Education [...]. And they made me grow and have a broader view on this division between the pedagogical and biodynamic areas. I ended up following the biodynamic area because I was a CNPq scientific research scholarship student in this field. And it was through contact with my supervisor that I ended up going on for my [...] Master's [...].

Interviewer: Do you have a Master's Degree in Physical Education?

Professor Rafael: Yes ... I have a Master's Degree in Physical Education.

Interviewer: So your Master's Degree was in Physical Education? Or do you have a Master's Degree in Biomechanics?

Professor Rafael: The line of research is "Biomechanics of Human Motion".

Interviewer: But what does your diploma say?

Professor Raphael: Master in "Biodynamics of Human Motion".

Bracht (2007) argues that the complex game of the existing forces in PE regarding titles that may attribute a status of Science to the discipline caused a new science to emerge - the Science of Human Kinetics or Human Motion from within its own field. In the reported case, despite the difficulty of a teacher to manage "two riverbanks", what we see is a specialist in Physical and Biological Sciences applying his knowledge to the PE field. Thus, we ask: How, in a teacher education course, should we deal with the inevitable application of knowledge from different fields in PE, in order to highlight it and open up prospects for renewed forms of access to knowledge and educational practice?

The second report reinforces our arguments and highlights the epistemological fragility of the field, as understood by an institution that was carrying out a public hiring examination process. The fact occurred in the year 2009 and was clearly seen in the selection process for federal primary school teachers at a particular federal military school located in the southeast of the country. There were openings for several fields, including for those with a Bachelor's Degree in Physical Education. The recommended study program 
was broad and included well-known authors who were relevant to the examination process. The impression was that it was intended to assess the contents in a balanced manner, by respecting the various PE subfields.

However, the examination was disappointing: of the forty questions on PE, none referred to pedagogical practices. Thus, we ask: Is it enough for a PE school teacher to know sports rules and biology only? How could we explain this point of view when the diploma required is that of a Licentiate? Is it not reasonable to assume that professional assessment would need to include pedagogical questions as related to the study program? Or did the examiners consider that this discipline involves knowledge of the body only? Or did they wish to select oblatu only? What teaching did they expect from those who were selected? In this case, what education should they have received from the universities?

Content relating to the field of Education was considered irrelevant and discredited. Why then should we include them in the program? The hegemonic side of the filed ended up causing a true "biological massacre" to PE.

The situation we depicted confirms the existing division in the profession. But, can we separate a specific activity from the broader set of activities within which it operates? Should we consider a PE teacher to be a sports coach or a trainer? Hughes points out two essential notions to understand the situation: diploma and mandate.

A license [diploma] is a legal authorization to exert certain activities that other people cannot do; a mandate is a legal obligation to ensure a specific function. [Diploma and mandate] are the bases for the "moral division of labor," which he [Hughes] defines as a process through which different functions valued by a group are distributed among its members, both groups and categories and individuals. An object of essential conflicts, this division of labor implies a hierarchy of functions and a division between core functions [...] and secondary functions [...]. (Hugues apud Dubar, 2005, p. 177-178)

Another aspect is shown and marks the ambiguous epistemological identity of PE: the secondarization of pedagogical knowledge, which ends up entailing discrimination of those professionals who value education and teaching. The focus on the body, on what is biological, on sports performance, eventually becomes hegemonic, resulting in the inability to treat the student as a whole. More subjective aspects of human motion are therefore disregarded which also affect the body (and results). As Dubar clarifies (2005, p. 180), "the entire profession tends to be formed in peer groups with their informal code, their selection rules, their interests and their common language, and to secrete professional stereotypes, excluding, in fact, those who do not correspond to them". 
The third case occurred at the same school. It was a pedagogical meeting addressing the theme "Sports and education: the school we have and the school we want". The central theme of the event made us believe we would see a debate on educational issues. However, what we saw was an uncompromising work in relation to Education.

The opening lecture at the Congress addressed sports only. The professional who spoke - a Military Officer - focused for almost two hours on his brilliant résumé of medals and the rigorous training he had gone through in a given sport before he won his gold medal at the Military World Games in July 2011. From the audience, we could clearly observe his body posture of "courage, patriotism and pride". He understood PE simply as a sport: it worked as a showcase for a strong, athletic and ready-for-war society. There was not, at any time, any concern for the educational side of PE, despite the event's proposal.

At a Congress on Education, what is the purpose of a speech with such characteristics? It highlighted the productive force of the body as an "athletic war machine." The distinguished officer spoke about the importance of sport in his life. Terms such as discipline, determination, love for the motherland, willpower and concentration were frequent in his speech. PE in its militaristic aspect was used as a method of behavioral education and for an individual's morale. Shaping man through body, to some, appears to be as efficient as through intellect.

Issues as noted in the Congress reinforced the view that, more than ever, the transfuga aspect of a teacher's identity construction is desirable. Integration between scientific, corporal and pedagogical cultures needs to be materialized. At the Congress, however, separations became evident and strengthened. Accepting the model of teaching extolled in the lecture, albeit implicitly, justified a lighter and restricted teacher education, not very prone to promote in a student the desire to learn more, self-reflection, criticism, search for innovation and alternatives, motion and body treatment by means of an integrated and consistent approach.

After the submission of the reports and our arguments, the epistemological fragility of the profession seems clear, along with the ambiguities involved in the construction of the identities for the teachers of the discipline, when they cross the different cultural spheres implied in this area of knowledge. These issues are reflected and develop in their teaching. In this process, more than oblatu or transfuga, more than hybrid identities, identities are many times built out of line, mismatched, frameless. It is important, however, that from the difficulties as expressed in everyday school life and in its not always positive features, we seek alternatives to another form of teaching education, where we can promote the construction of transfuga identities, foster a self-reflection process and the possibility to transform PE into a third degree learning space, as suggested by Bauman (2008). 


\section{Final Considerations}

The current hegemonic patterns in the teaching of PE (competitive and biological) emerged in Modernity. Such hegemony can be linked to values and excesses of a modern industrial capitalism. Its use, as a dominant form of PE practice in the school context, implies reducing and distorting the educational function of a teacher, which implies developing a historical view and a critique of society and the world in the student. This approach would contribute to a more independent construction of identities that could go against the grain of the models preferred by the current social order.

In this context, we argue that progressive PE teaching paths are those aimed at destabilizing the technical/biological paradigm still present in the field. For these trends, the current features of the body culture of motion in contemporary schools are still, unfortunately, used to produce a "pseudoawareness" in the student and, even more so, to stimulate the uncritical consumption of a cultural industry that focuses on certain aesthetic and body patterns. It ends by forming oblatus, unable to build bridges and cause the confrontation of different cultural spheres. It reveals the inability to produce alternatives that would question those habits acquired in the school process and enhance the importance of framing and accepting these habits less and less as inevitable.

Bracht (1999) adds that the use of a new theoretical referential to understand human motion depends on the change of social imaginary about the body and its practices. It depends on overcoming the mind/body dichotomy. It depends on integrating body education within the broader education level of the individual, which demands the contribution from both science and pedagogical theorizations and disciplines. It depends on renewed and creative teacher's work and education.

This change in the imaginary, which is expected to become evident at work and in teacher education, can be characterized by:

1. Admission - by the professionals that the epistemological identity of $\mathrm{PE}$ is an unclear process, with not very clear paths, to be continuously questioned and reformulated;

2. Promotion of a teacher education process to develop selfeducation and critical professionals;

3. An attempt to give greater curricular balance in education, which requires a greater number of disciplines from the Social Sciences and Humanities in the PE curriculum;

4. Change in teaching, which needs to be guided by the perspective of a second and third degree learning, which expresses and inspires education in which the future teacher is the central character in the process;

5. New forms of management which, more than results-, good performance- and efficiency-oriented, may strengthen teacher 
professionalism, seeking autonomous, creative and critical teaching;

6. Enhancement of teachers' work, which should be seen not as a secondary occupation in relation to material work, but rather as one of the keys to transforming contemporary societies (Tardif \& Lessard, 2005).

At the same time, it is essential to decrease the distance between the theoretical production in PE and the teaching practice effective in educational institutions. Existing gaps in the University/School relationship seem to contribute to the distortion of the epistemological reality in PE. Although the family, in general, continues to relate PE to sports and Olympism, the academic speech in the field seems, in part, to have overcome this limitation. The school continues, however, tending to follow the family concept. Oblatus insist on separating the mind from the body, science from its application, PE from the pedagogical knowledge that may guide it.

It also seems essential that teachers at schools seek more aggressiveness in their classes, by giving up acquired hegemonic sports practices and habits and promoting pedagogical activities and alternatives in PE teaching. We argue that the autonomy and the authority for the creation of a "new physical education", according to their specific contexts, are better than the assurance of a true "PE", as was the case in the early days of Modernity. This does not mean abandoning the need to support the existing education conceptions present in these practices, but performing them democratically, assuming the foundations that underpin its own weakness. In other words, we argue a new PE guided by what Bauman calls second and third degree learning. Let us then finish with his words:

In a world where no one can (although many do, with consequences ranging from irrelevant to disastrous) anticipate the type of expertise that will be needed tomorrow, the debates that may require mediation and the beliefs that may require interpretation, the recognition of many and varied shapes and canons of higher learning is the sine qua non condition for a university system that is able to oppose the postmodern challenge. (2008, p. 176)

\section{References}

Assunção, A. \& Oliveira, D. (2009). Intensification of work and health of teachers. Education \& Society, Campinas, 30(107): 349-372.

Bauman, Z. (2008). The individualised society: lives counted and stories lived. Rio de Janeiro: Jorge Zahar.

Bracht, V. (1999). The Constitution of the pedagogical theories of physical education. The contract Cedes, Campinas, 19(48): 69-88. 
Bracht, V. (2007). Physical education \& science: scenes from a marriage (UN) happy. $3^{\mathrm{a}}$ ed. Ijuí, RS: Unijuí.

Brazil. (1997). The Ministry of education. Fundamental Education Department. National curricular parameters: introduction to national curriculum parameters. Brasilia: MEC/SEF.

Foucault, M. (2004). Monitor and punish: the birth of the prison. $29^{\mathrm{a}}$ ed. Petrópolis, RJ: Voices.

Hall, S. (2006). Cultural identity in Postmodernity. $11^{\mathrm{a}}$ ed. Rio de Janeiro: DP \& A.

Hugues apud Dubar, C. (2005, p. 177-178). Socialization: building social and professional identities. São Paulo: Martins Fontes

Tardif, M. \& Lessard, C. (2005). The teaching job: elements for a theory of teaching as a profession of human interactions. Petrópolis, RJ: Vozes.

Vargas, C. P. (2010). Identities in disarray: the physical educator's ambivalence in the crisis of modernity. Dissertation (master of education) - Catholic University of Petrópolis.

Vieira, R. (2009). Personal Identity: interactions, possibility and cultural metamorphosis. Lisbon: Colibri. 
